\title{
LA INVESTIGACIÓN SOBRE LA ADMINISTRACIÓN EN COLOMBIA (1965-2015): BALANCE Y PERSPECTIVAS
}

\author{
Florentino Malaver Rodríguez"*: \\ Francisco López Gallego ${ }^{* * *}$
}

* doi: 10.11144/Javeriana.cao29-52.iacb. El artículo se recibió el 15/05/2016 y se aprobó el 20/06/2016. Sugerencia de citación: Malaver, F. y López, F. (2016). La investigación sobre la administración en Colombia (1965-2015): balance y perspectivas. Cuadernos de Administración, 29 (52), 141-166. http://dx.doi. org/10.11144/Javeriana.cao29-52.iacb

** $\mathrm{PhD}(\mathrm{c})$ en Competitividad Empresarial y Desarrollo Económico, Universidad de Deusto, España. Magister en Economía de la Universidad Nacional de Colombia. Profesor Asociado de la Facultad de Ciencias Económicas y Administrativas, Pontificia Universidad Javeriana, Bogotá, Colombia.

Correo electrónico: fmalaver@javeriana.edu.co

*** Ph.D. Ciencias de la Gestión, ESADE, Barcelona. Profesor titular Universidad EAFIT, Medellín, Colombia. Correo electrónico: flopez@eafit.edu.co 


\section{La investigación sobre la administración en Colombia (1965-2015): balance y perspectivas}

\section{RESUMEN}

Tras cinco décadas y media de creación de las facultades colombianas de administración (FCA) y luego de un tardío y lento desarrollo de la investigación, en los últimos 15 años se han registrado profundos cambios que produjeron su institucionalización y despegue y, otros, como la internacionalización de la publicación de sus resultados y la creación de una oleada de doctorados, están en ciernes. Apoyado en los estudios colombianos y la discusión internacional sobre el tema, este artículo propone una periodización de su trayectoria y una interpretación de las fuerzas y factores que definieron su ritmo y orientación en cada periodo, para brindar elementos que contribuyan a una mejor comprensión del pasado y presente de la investigación, y a mostrar los desafíos y alternativas que enfrentan las FCA para consolidar los procesos en curso.

Palabras clave: investigación en administración, Colombia, doctorados, internacionalización.

Clasificación JEL: M19

\section{Research on management in Colombia (1965-2015): Balance and perspectives}

\section{ABSTRACT}

After five and a half decades since the creation of the Colombian management faculties (FCA) and after a late and slow development of research, profound changes have been registered in the past 15 years that produced its institutionalization and beginnings and, others, such as the internationalization of the publication of its results and the creation of a series of doctorates are in their infancy. Supported by the Colombian studies and the international discussion on the subject, this article proposes a chronological framework of its path and an interpretation of the forces and factors that defined its rhythm and orientation in each period to provide elements that can contribute to a better comprehension of the past and present of research, and to show the challenges and alternatives that the FCA face to consolidate the processes that are currently underway.

Keywords: Research on management, Colombia, doctorates, internationalization

JEL Classification: M19

\section{A pesquisa sobre a administração na Colômbia (1965-2015): balanço e perspectivas}

\section{ResUMO}

Depois de cinco décadas e meia de criação das faculdades colombianas de administração (FCA) e depois de um tardio e lento desenvolvimento da pesquisa, nos últimos 15 anos registraram-se profundas mudanças que produziram sua institucionalização e desdobramento e, outros, como a internacionalização da publicação de seus resultados e da criação de uma onda de doutorados, que estão iniciando. Apoiado nos estudos colombianos e na discussão internacional sobre o tema, este artigo propõe uma periodização de sua trajetória e uma interpretação das forças e fatores que definiram seu ritmo e orientação em cada periodo, para fornecer elementos que contribuam a um melhor entendimento do passado e presente da pesquisa, e a mostrar os desafios e alternativas que enfrentam as FCA para consolidar os processos em curso.

Palavras-chave: pesquisa em administração, Colômbia, doutorados, internacionalização. Classificação JEL: M19 


\section{Introducción}

La investigación en el campo de la administración en Colombia vive una coyuntura excepcional. Luego de constatarse por tres décadas su escasez y lento crecimiento, y de reiterarse el diagnóstico de su estado incipiente (Dávila, 1980; Rodríguez, Dávila y Romero, 1990; Malaver, 1999), en la última década se registró su despegue (Malaver 2006), la convergencia de fuerzas que impulsan la internacionalización de sus publicaciones (Orozco y Villaveces, 2015; Malaver, 2016) y una "oleada" de creación de programas de doctorado (Calderón et al., 2010), que sin duda marcan cambios profundos en su trayectoria. Sin embargo, la consolidación de esos procesos, así como el sentido y alcance de su impacto en el futuro de la investigación dependerá, en mucho, de las orientaciones y decisiones que se tomen en esta coyuntura.

Por ello, el objetivo de este artículo es proporcionar elementos que contribuyan a estructurar una visión amplia -contextualizada- y de largo plazo de la investigación sobre la administración en Colombia y, de ese modo, para tener una perspectiva más comprensiva y estratégica de la coyuntura actual, así como también facilitar la consolidación de su institucionalización y de procesos tan estratégicos como la internacionalización de sus publicaciones y sus doctorados, que están en curso.

Para ello se presenta, en síntesis, una reconstrucción de la trayectoria de la investigación, apoyada en los estudios sobre la investigación en gestión en el país. Estudios que, como en pocas temáticas en el ámbito de la administración en Colombia, permiten seguir esa trayectoria ${ }^{1}$. Para esa reconstrucción se propone una periodización que permite identificar los rasgos que caracterizan cada una de las etapas de esa trayectoria; se identifican los factores - del entorno y de las propias facultades colombianas de administración (FCA)con mayor incidencia en su dinámica en cada etapa; y se ahonda en la inserción de la investigación en las FCA y su contribución al conocimiento y/o al desarrollo del país. AL final, se hace un balance sobre lo que ha cambiado y lo que permanece $y$, adicionalmente, se vislumbran los mayores retos para el futuro más inmediato.

1 Entre ellos se encuentran los artículos que hacen parte de este Especial de Cuadernos de Administración, que aportan evidencias para la interpretación de los cambios que están dándose en el presente y avizorar los retos para el avance de la investigación. 


\section{Periodo 1965-1995: de los inicios al estado incipiente de la investigación}

El trabajo de Mayor (1990) sobre la profesionalización de la administración en Colombia, ayuda a entender el comienzo de la evolución de la investigación en las FCA. La administración ingresó por vía de los ingenieros, que en la Escuela Nacional de Minas de Medellín, empezaron a enseñar a Taylor en 1912 y a Fayol en 1916 -en Harvard iniciaron hacia 1918-; luego por los abogados en Bogotá, que en 1931 comenzaron a enseñar administración a los estudiantes de derecho de la Universidad Javeriana; más tarde por la Escuela de Administración Industrial del Gimnasio Moderno, que en los años 50 tituló a los primeros economistas - administradores y usó el método de casos de Harvard; y solo en los años 60 se crean las primeras FCA y se titulan los primeros administradores profesionales ${ }^{2}$. Y de ello se "heredó" una alta sustituibilidad en el mercado laboral con quienes primero enseñaron y desempeñaron el oficio -ingenieros, abogados y economistas-, $y$ “ un fuerte acento anti-intelectual vía ingeniería, que es quizá la menos intelectual de las profesiones" (Mayor, 1990, p. 106) que, como veremos, dificultaría el avance de la investigación en las FCA y el trabajo de los primeros investigadores.

Son precisamente dos de esos investigadores quienes publican trabajos pioneros sobre el estado de la investigación de la administración en Colombia, señalando que a comienzos de los años 80 esta es casi inexistente (Dávila, 1980, 1982; Ogliastri, 1982), mientras estudios de comienzos y fines de los 90 diagnostican que su estado es incipiente (Rodríguez, Dávila y Romero, 1990, 1992; Malaver, 1999; Malaver, Romero, Cortés, Ruiz, Perdomo y Peralta, 2000).

Los estudios mostraron la "escasez extrema" de investigación hasta comienzos de los 80 (Rodríguez et al., 1990), como quiera que, pese a los aumentos registrados desde 1985, entre 1990 y 1998 se publicaron el 64\% de los libros, el $82 \%$ de los capítulos en libros, y el $60 \%$ de los artículos en revistas académicas, de todos los publicados en el periodo comprendido entre 1965 y 1998 (Malaver, 1999). A pesar de esto, se diagnosticaba que a finales de los 90 las publicaciones de las FCA eran muy escasas; se concentraban en cuatro facultades y en menor grado en otras siete, pero era tan baja, que si cada uno de los profesores de estas once facultades hubiese publicado un artículo cada cuatro años entre 1990 y 1997, se habría superado el número de publicaciones efectuadas en los 33

2 En Eafit se crea en 1960. En 1966 existían siete facultades de administración, y diez años después se tenían 35 (Dávila, 1988; López, 2001). 
años estudiados 3 ; y las publicaciones de la Facultad de la Universidad de los Andes superaba las hechas por las restantes 68 facultades; el $88 \%$ de los investigadores registraban máximo dos publicaciones, que poco dialogaban con los trabajos previos, generándose una protuberante fragmentación. Además, su calidad era muy heterogénea, según lo revelaban los artículos publicados en revistas académicas nacionales indexadas, que como máximo publicaban un artículo de investigación cada dos fascículos (Malaver, 1999).

El poco avance de la investigación en este periodo encuentra buena parte de su explicación en su débil inserción en las FCA. Una de las causas principales -la más mencionada- de esto, era la existencia de un ambiente "anti-intelectual" en las facultades que dificultaba reconocer y aceptar la importancia de la investigación y generaba condiciones adversas para su institucionalización (Mayor, 1990; Dávila, 1988; Ogliastri, 1982). Se reitera que la aversión a la teoría y a "Los teóricos" generaba hostilidad hacia los investigadores (Dávila, 1982, 1988) quienes, en ese contexto, se convertían en "huéspedes incomodos" en sus Facultades. Esto se traducía en poca comprensión de las lógicas y dinámicas de la investigación; en desatención y desinterés de las facultades por la investigación; en la tardía incorporación de la figura del investigador ${ }^{4}$ y en la escasez de profesores con formación y entrenamiento en investigación. Tales condiciones llevaban a manifestar que ante la falta de "colegas críticos, de una comunidad científica, comparto el sentimiento de varios investigadores que dirigen su trabajo y publicaciones a posibles colegas de una próxima generación, hacia el futuro" (Ogliastri, 1982, p. 127).

Esta situación afectaba el cumplimiento del papel de la investigación en la docencia, que se consideraba la base de una educación de calidad, una de las principales preocupaciones de los investigadores en estos años. Por ello se planteaba que se debía superar la falsa dicotomía entre teoría y práctica, pues lo teórico tiene sentido en la medida en que explica la práctica; que acercar a los estudiantes a la realidad administrativa significaba hacerlo con rigor: investigarla; y que si la docencia no tenía como base la

3 Mediante un inventario de las investigaciones publicadas entre 1995 y 1998 sobre la administración en Colombia (Malaver, Romero, Cortés, Ruíz, Perdomo y Peralta, 2000).

4 El reconocimiento de la figura del investigador en administración, esto es, una persona dedicada de tiempo completo a investigar, es tardía, ubicándose en los años 80 (Mayor, 1990). Estos provenían en su mayoría de disciplinas vecinas de la administración -como la economía, la psicología, la sociología-, a las que tenían que acudir en la indagación en cualquiera de las áreas de la administración en busca de explicación, pues las "teorías administrativas" como el taylorismo, el fayolismo o las relaciones humanas, no tenían el "estatuto de teorías científicas" (Mayor, 1990; Ogliastri, 1982; Dávila, 1982), y se citaba el enfoque fayolista, considerado como una "ciencia administrativa", autoproclamado universalista, del cual Simon (1965) -citado por Dávila (1988)-, había mostrado que sus 'principios' "no eran más que proverbios”. 
actividad investigativa era deficiente, pues sin ella era repetitiva, carente de rigor y de carácter crítico (Dávila, 1982, 1991). Por ello, se indicaba que las FCA debían tener como un propósito fundamental estudiar la realidad administrativa del país, ya que sin "una disposición hacia escudriñar realidades complejas, en vez de solucionar problemas de texto con respuesta y recetario, las facultades de administración se limitan a transferir mecánicamente, tecnologías administrativas, muchas veces inadecuadas para nuestro medio o que requieren de una tarea de adaptación" (Dávila, 1991, p. 50).

En ese sentido, se argumentaba que la escasez de investigación, su carácter individual y su presencia marginal en las FCA, contribuyó a la "crisis en la calidad de la educación gerencial" ofrecida en el país; y a la proliferación de una educación profesionalizante, centrada en las técnicas y modas administrativas que, por lo demás, nutría el ambiente anti-intelectual y hostil hacia la teoría (Rodríguez et al., 1990, 1992; Dávila, 1991, 2005).

Si el diagnóstico era de una "crisis de calidad", ¿qué sostenía la masificación de ese tipo de educación, revelada por el progresivo crecimiento de los programas de administración? Por una parte, por el crecimiento de la demanda de administradores profesionales, ocasionado por procesos que veremos más adelante; por otra parte, por el hecho -señalado por un investigador- de que en el ámbito de la administración "las facultades pueden estar bien posicionadas sin necesidad de investigar" (Malaver, 1999); realidad confirmada por la revista Dinero (2000) al señalar "que el mercado y la academia no siempre coinciden", refiriéndose a los resultados del "primer ranking de administración efectuado en Colombia", en el que aparecen bien posicionadas Facultades con muy poca o ninguna investigación.

El poco desarrollo de la investigación en este periodo también incidió negativamente en la generación de conocimiento para comprender la realidad administrativa, en un país que necesitaba, cada vez más, empresas mejor gerenciadas. Asunto que constituía otra de las preocupaciones centrales de los investigadores en ese periodo, y que estaba fundamentado en la concepción de que la administración es una práctica social con especificidad temporal y espacial (Dávila, 1991) y que, en consecuencia, su problemática y las necesidades de conocimiento están asociadas al contexto específico en cuestión.

De hecho, el proceso de industrialización, según Mayor (1990), generó la necesidad de administradores que soportó la creación de las FCA. Luego, por las políticas proteccionistas, las empresas no tuvieron que fortalecer su eficiencia y competitividad para tener un relativo éxito económico y, por ello, tampoco demandaban generación de conocimiento 
en las FCA. Pero esa situación - de empate negativo- cambió en los años 90 a raíz de la apertura económica, que obligó a las empresas a competir con mayor eficiencia, capacidades gerenciales y estratégicas y que, entonces, se vieron forzadas a acudir en búsqueda de respuestas en las facultades de administración (Dávila, 2005; Malaver, 1998).

Pese a los aumentos registrados en los años 90, la investigación fue escasa y, además, su débil conexión con problemáticas, discusiones y políticas públicas estratégicas aportaba síntomas de irrelevancia (Malaver, 1999; Malaver et al., 2000). La respuesta de las FCA se centró en la oferta masiva de cursos de posgrado y educación continua. Y el vacío de conocimiento fue copado por gurúes y best sellers propagadores de "modas administrativas", vendidas como panaceas para los problemas organizacionales. Estas se asumían de manera acrítica y su aplicación era ineficaz (López, 1998), pero generaban la percepción de que se estaban brindando e incorporando los avances más recientes y novedosos del conocimiento administrativo.

Finalmente, la perspectiva internacional en la mirada de la investigación fue, de alguna manera, contradictoria. Si bien se hacía referencia a autores e informes internacionales -como el de Porter y McKibbin (Dávila, 1990)-, había un acentuado énfasis en las problemáticas de nuestra realidad. Pero, a partir de considerar la administración como práctica social con especificidades contextuales, y de criticar el carácter ahistórico y etnocentrista de la pretendida universalidad de muchos textos-que implicaba transferirlos y aplicarlos sin cambios en los países receptores-, se llegó a proponer la búsqueda de "una administración para América Latina" (Echeverry, Chanlat y Dávila, 1990). Esto significaba, identificar sus características distintivas, reflejadas en las prácticas y la teorización administrativa y buscar nuevas formas de hacer y pensar la administración (Chanlat, Echeverry y Dávila, 1990).

Mientras tanto, en el ámbito internacional se estaban viviendo los resultados producidos por un cambio dramático basado en los informes - de 1959- de las Fundaciones Ford (Gordon y Howell, 1959) y Carnegie (Pierson, 1959). Este significó un giro de las Facultades orientadas hacia el ámbito práctico durante la primera mitad del siglo XX -responsable de su bajo estatus académico (Mintzberg, 2005), pues se criticaba que el conocimiento administrativo y organizacional era básicamente "una colección de anécdotas y casos de estudio" (Corley y Gioia, 2011); ahora se orientaban hacia la excelencia, derivada del énfasis en el rigor académico resultante de asumir el modelo de las ciencias naturales y la teoría económica -neoclásica-, que privilegiaba el método hipotético-deductivo y el rigor -cuantitativo- para adelantar una investigación centrada en el desarrollo discipli- 
nar (Hambrick, 2007). Ese giro se vio favorecido por la acelerada expansión económica registrada en los países desarrollados, lo que propició el surgimiento de una amplia variedad de teorías y desarrollos disciplinares entre los años 70 y 80 , divulgada a través de los journals académicos (Colquit y Zapata, 2007).

De ese modo, en este periodo más allá de las obvias diferencias en el nivel de desarrollo de la investigación, tanto las visiones y las condiciones existentes la impulsaban en distintas direcciones y ritmos en los países desarrollados y en Colombia. Allá la investigación avanzaba propulsada por la legitimación que le otorgaba el énfasis en lo disciplinar y en el rigor como fuente de excelencia, mientras aquí el énfasis en lo profesional y en las técnicas y modas administrativas contribuía a debilitar la frágil situación de la investigación. Allá se consideraba que lo clave era su contribución a la teoría, mientras aquí -motu proprio-se propugnaba por una investigación empírica, orientada principalmente a contribuir a solucionar los problemas empresariales, aunque con resultados en los que se advertían atisbos de irrelevancia (Malaver, 1999).

\section{Periodo 1996-2005: institucionalización y despegue de la investigación}

Pese a las mejoras registradas en los años 90 , persistían muchas de las condiciones que dificultaban la investigación y no se vislumbraban fuerzas al interior de las FCA capaces de impulsar transformaciones que rompieran las inercias que conducían al lento avance de la investigación; por ello, a comienzos de la primera década de los 2000 habían fundadas dudas acerca de si el desarrollo de la investigación en las FCA era posible o "una utopía" (Dávila, 2005). Inesperadamente, la política pública fue el desencadenante de esas transformaciones. En efecto, desde mediados de la segunda parte de la década de los 90 se gestaron cambios inducidos por las políticas de ciencia, tecnología e innovación (CTI), que crearon condiciones favorables para la investigación en las FCA y provocaron avances que significaron una ruptura en la trayectoria hasta entonces registrada (Malaver, 2006).

Los estudios sobre la investigación en gestión captaron progresivamente los cambios ocurridos entre fines de los 90 y comienzos de la década de 2000, y que el factor que los impulsó fue la política de CTI. De hecho, en Malaver et al. (1999) ya se registraban las convocatorias de Colciencias - para grupos de investigación y revistas académicas- de 1996 y 1997, y en Calderón y Amaya (2005) y Dávila (2005) se analizaban los resultados de las convocatorias de grupos. Pero es en el trabajo de Malaver (2006) que se analiza la política y su impacto en la investigación en las FCA. 
En ese trabajo se detallan los distintos instrumentos y mecanismos (tecnológicos, normativos y de incentivos) de las políticas de CTI y de educación que indujeron la institucionalización de la actividad investigativa en las FCA, reflejada en el aumento en el número de investigadores y de grupos de investigación, de las investigaciones, de las publicaciones en libros y capítulos de libro y, en particular, de los artículos en revistas -sobre todo nacionales- indexadas internacionalmente (Malaver, 2006). La generalización del proceso y los avances registrados entre 1996 y 2005 permitían pensar, en síntesis, que en ese periodo se produjo el despegue de la investigación y que esto significó un quiebre histórico. Al respecto se indicaba que:

La expansión registrada en el periodo 2000-2006 (...) cambió el panorama de la investigación existente a fines de la década de los noventa: esta ya no es una actividad concentrada en unas pocas universidades $y$, dentro de estas, en un núcleo minoritario de profesores que la realizan de manera individual y aislada. Hoy la adelantan grupos de investigación localizados en muchas facultades de administración y con avances en la cantidad de grupos, en sus categorías, en el volumen de investigación y consultoría, en la cantidad y calidad de la producción, que indican que estamos en presencia de un importante desarrollo (institucionalización) y de la emergencia de nuevos liderazgos investigativos. (Malaver, 2006, pp. 105-106)

Trabajos posteriores -como los de Calderón et al. (2010) y Calderón et al. (2014)- confirmaron la fuerza del proceso de institucionalización y el avance de la investigación. Esto incidió en una mirada tan positiva de los avances alcanzados que -apoyados en los criterios de evaluación de grupos e investigadores de Colciencias- se llegó a plantear que al comienzo de la década actual el país ya contaba con grupos de investigación de "élite" en sus FCA (Calderón et al., 2014).

Por lo visto, la influencia de la política pública fue tan preponderante en este periodo (1996-2005) que también impregnó los estudios sobre la investigación en gestión en Colombia. De ese modo, los principales referentes para el análisis y la evaluación de la actividad investigativa y de sus resultados eran nacionales. Pero en los criterios de evaluación de Colciencias estaban aplicados, implícitamente, estándares internacionales, por ejemplo -en el Publindex-, para las revistas en que se publicaba; que en estos años eran principalmente las revistas nacionales indexadas. Así, aunque mediada y tácita, la internacionalización de la investigación estaba presente. 
Aunque en ciernes todavía, los criterios de evaluación de Colciencias apuntan hacia el modelo de investigación de las ciencias básicas - lo que se haría evidente años después-. Este, que fue el modelo impulsado en el campo de la administración por más de cuatro décadas, comienza a ser cuestionado en el ámbito internacional desde los años 2000. Se señala que al privilegiar el rigor sobre la práctica, las escuelas de negocios "perdieron el rumbo" (Bennis y 0'Toole, 2005), la relevancia y la legitimidad en el mundo empresarial (Pfeffer y Fong, 2001; Mintzberg, 2005; Augier y March, 2007). Con respecto a la investigación, a las críticas académicas sobre la irrelevancia de mucha de la investigación publicada se sumó la presión de los financiadores -como el gobierno británico- por su incidencia sobre la práctica (Hambrick, 2007; Bartunek y Rynes, 2014).

En síntesis, bajo el influjo de la política pública se institucionaliza la investigación en las FCA y se comienzan a aplicar estándares que parecen orientarla en una dirección que empieza a ser cuestionada internacionalmente en el ámbito del management. En esas críticas está explícita la demanda de una investigación más relevante, que en el periodo anterior fue una preocupación central de los estudios sobre la investigación en las FCA, pero que en este periodo apenas apareció entre bastidores.

\section{Periodo 2006-2015: presión institucional y perspectivas de consolidación}

Impulsados por una fuerte "presión institucional" y la lucha entre las principales FCA por un mejor posicionamiento académico (Orozco y Villaveces, 2015; Orozco, 2015), en el periodo comprendido entre 2006 y 2015 se han producido cambios en materia de la investigación en las FCA tanto o más profundos que los registrados en la década anterior. Entre ellos se destacan dos procesos igualmente acelerados: la internacionalización de las publicaciones (Malaver, 2016) y la creación de programas de doctorado (Orozco, 2016). Estos procesos han contribuido a profundizar la institucionalización al interior de las FCA, y están llamados a constituirse en las puntas de lanza de una previsible consolidación de la investigación en las FCA. Sin embargo, están impulsados por fuerzas contradictorias y generan tensiones y desafíos que, aunque en curso, es necesario dilucidar, pues de su comprensión y tratamiento dependerá el futuro de la investigación en este campo del conocimiento en Colombia. Por ello, aquí se abordará la problemática asociada a su avance -apoyándose mucho- en los artículos que hacen parte de este Especial de Cuadernos de Administración. 


\subsection{La internacionalización inducida de la publicación de las FCA}

En estos años, tal como se señala en el artículo de Malaver (2016) -en este especial y en el que se basa este acápite- la política de Colciencias avanzó en la aplicación de exigentes estándares internacionales para evaluar las publicaciones de los investigadores y grupos de investigación colombianos. En el campo de la administración esto se sumó a las exigencias de las acreditadoras internacionales -a las que acuden en grado creciente las FCA - y la búsqueda de una mejor clasificación en los rankings internacionales, configurando una fuerte presión institucional para aumentar la cantidad y calidad de las publicaciones internacionales (Orozco y Villaveces, 2015). Esto aconteció, pero en tal magnitud que se produjo un nuevo -segundo- quiebre histórico en la trayectoria de la investigación en las FCA (Malaver, 2016).

La incorporación acelerada de los más exigentes estándares internacionales de evaluación para los artículos, hecha por Colciencias y los rankings y las acreditadoras, con base en la clasificación de los journals donde se publica ${ }^{5}$-como indicador de calidad de los artículos producidos- y de las citaciones -como indicador de su influencia-, tiene efectos positivos (Malaver, 2016). De hecho, el aumento simultáneo en la cantidad de artículos publicados en los journals incluidos en la WoS y Scopus y en los journals de más alta clasificación revela una apropiación cada vez mayor de la literatura internacional más importante, mejoras sustanciales en la calidad del conocimiento generado y aceleradas curvas de aprendizaje sobre las lógicas de la publicación y circulación del conocimiento al nivel internacional.

Pero, a renglón seguido, se aprovecha el despegue e internacionalización tardíos de la investigación en las FCA para derivar lecciones de la experiencia internacional en el ámbito del management que permiten identificar sesgos y problemas derivados de las presiones institucionales, que podrían superarse. Igualmente, se identifican desafíos y tensiones derivados de las contradicciones de los procesos en curso que son insuperables, pero que podrían enfrentarse de manera creativa, de modo que posibiliten un posicionamiento académico internacional concordante con mayores niveles de legitimación social.

5 La clasificación de los Journals, como Q1, Q2, Q3, Q4 es determinado por el "factor de impacto" el cual, a su vez, depende de la citación de los artículos publicados, criterio extendido por el "índice h". Esto está analizado con detalle en el artículo de Malaver (2016). 
En tal sentido, en el artículo de Malaver (2016) se advierte que la evaluación basada en la citación orienta la investigación y las publicaciones hacia el modelo implantado cinco décadas atrás en los países desarrollados en el ámbito del management. Este era objeto de críticas a comienzos del siglo XXI, que arreciaron a raíz del papel de las escuelas de negocios en la reciente crisis en los países desarrollados -que develó, por ejemplo, severos problemas éticos de algunos de sus egresados- por su sesgo en favor del rigor y en detrimento de la relevancia -utilidad social- del conocimiento generado. Tales críticas han conducido a replanteamientos en el ámbito institucional que buscan mayor equilibrio entre el rigor -la teoría- y relevancia -el desarrollo de habilidades- en los currículos de pregrado (AACSB, 2012) y en la composición de la planta de profesores de las escuelas de negocios.

La discusión ha sido más profusa en lo relacionado con la investigación y su publicación. Incluso desde la perspectiva puramente académica. Al respecto, se indica que en el modelo se tienden a valorar los artículos por su contribución a la teoría, pero dentro de un determinado paradigma -al que está adscrito el journal donde se publica-, y de ello se generan lógicas que son responsables, por un lado, de la poca originalidad de los artículos, entre otras razones, por la poca probabilidad de que cuando el paradigma está maduro surja un conocimiento sorprendente, interesante y que conduzca a un nuevo entendimiento de su objeto de estudio (Kuhn, 1962; Alvesson y Sandberg, 2013) y, por otro, de un marcado sesgo hacia la monodisciplinariedad que caracteriza a los journals top del management y que dificulta la interdisciplinariedad consustancial a este campo del saber (Rafols et al., 2012).

Como era de esperarse en el marco de la crítica al modelo, la discusión se ha centrado en la irrelevancia y en la reducción de sus sesgos. En este sentido se propone valorar los textos por su contribución a la teoría y su utilidad social (Corley y Gioia, 2011), dar tanto valor a los enfoques hipotético-deductivos como a los inductivos, y a los métodos cuantitativos como a los cualitativos ${ }^{6} \mathrm{y}$, sobre todo, tender puentes entre académicos y practicioner pero reconociendo que sus visiones e intereses no solo son distintos sino que sus diferencias son insuperables, y que por lo tanto los esfuerzos deben orientarse a manejarlos como fuente de oportunidades para generar alternativas novedosas de investigación (Bartunek y Rynes, 2014)․

6 Esta cuestión es tratada con mayor detalle en los textos mencionado de Malaver (2016) y Martínez y Páez (2016), en particular, en lo relacionado con los modos (uno y dos) de producir conocimiento; también se aborda en el artículo de 0campo, Gentilin y Gonzales (2016). Todos ellos hacen parte de este número Especial.

$7 \quad$ Estas discusiones y alternativas que son presentadas con algún detalle en los artículos de Malaver (2016) y Martínez y Páez (2016) del Especial, así como también en Orozco (2015) y en Orozco y Villaveces (2015). 
Esos replanteamientos han alcanzado a journals norteamericanos (Academy of Management Journal, Academy of Management Review, Strategic Management Journal) y europeos (Journal of Management Studies, British Journal of Management), que asumen las críticas en torno a la poca relevancia y originalidad de muchos artículos publicados. Entonces reconocen la necesidad de ampliar los criterios para evaluar los artículos por su contribución a solucionar problemas empíricos relevantes (George, 2014), de revalorizar la construcción de la teoría por vía inductiva y métodos cualitativos, de propiciar los diálogos entre distintos actores.

En ese sentido, en el artículo de Malaver (2016) se señala que la internacionalización (geográfica) de las contribuciones (autores), ha dado lugar en algunos "journals top" a los gérmenes de mayor diálogo académicos entre países emergentes y desarrollados. Estos surgen de reconocer que las diferencias en los niveles de desarrollo, culturas, valores, visiones e instituciones, se expresan en una rica variedad de prácticas y problemas administrativos, que para explicarlos y solucionarlos pueden requerir de nuevos conceptos o teorías, o de ampliar los existentes (Barkema et al., 2015). En este caso, la lógica del modelo imperante -del estado del arte e identificación del "vacío" a llenar para contribuir a la teoría- resulta insuficiente, pues de partida se requiere conocer a profundidad el contexto propio, para evaluar la capacidad del conocimiento paradigmático existente con el fin de explicarlo, o si debe adaptarse o recrearse para ello; o pueden encontrarse problemas relevantes o emergentes y específicos de ese contexto que a poco andar muestren que para explicarlos es necesario crear conocimiento: nuevo y original. Esto significa que desde esos journals se está ventilando y proponiendo, la posibilidad de aprovechar la amplia variedad de realidades y problemáticas que requieren de explicaciones en el origen de conocimientos que enriquezcan el existente, es decir, planteando posibilidades de ampliar el conocimiento existente. $Y$ esto es lo que se ha pedido reiterada e infructuosamente, por varios de los autores aquíabordados, desde los trabajos pioneros. Sin embargo, como se discute con detalle en Malaver (2016) enfrenta sus mayores obstáculos en el ámbito de la publicación, y como veremos adelante, en la lógica aquí discutida y que hoy parece imponerse en las FCA.

En síntesis, una mirada a la discusión internacional sobre la valoración de la investigación en management muestra que, irónicamente, si aplican los estándares internacionales de evaluación aplicados por Colciencias y las demás fuerzas que configuran la presión institucional, y que hoy tiende a servir de rasero para evaluar las publicaciones de las FCA, se impulsaría la investigación en una dirección opuesta a las tendencias internacionales actuales, se desconocerían las características intrínsecas del campo del conocimiento, y 
se privilegiaría la contribución a la teoría de un modo que desincentivaría la investigación enfocada a la contribución social. De esa discusión surge una conclusión insoslayable: se deben asumir los estándares aplicados por Colciencias para evaluar la investigación, pero deben transcenderse para poder contar con unos que respondan a las exigencias actuales de la investigación en management. Y esto implica, como se plantea en el artículo de Malaver (2016) desarrollar y aplicar indicadores que también midan el impacto de las publicaciones -los artículos- por su relevancia-utilidad social-. Esa mirada también muestra la vigencia internacional del propósito de convertir la propia realidad en fuente de conocimiento relevante y original y de ampliación de la teoría existente ${ }^{8}$.

\subsection{La creación de doctorados en las FCA}

La "oleada" de creación de programas de doctorado de administración entre 2004 y 2015 marcó un hito en la historia de la administración en Colombia. Este proceso también fue propiciado por la política pública y la búsqueda de acreditación internacional por parte de las FCA, se retroalimenta con la internacionalización de las publicaciones para profundizar la institucionalización de la investigación en las FCA y facilitar el que puede ser el cambio más profundo y significativo en la vida de las facultades: el paso de la profesionalización a la disciplinarización de la administración en el país -como lo han denominado Sanabria, Saavedra y Smida (2014)-. No obstante, el grado y sentido en que este proceso puede incidir en la dinámica de la investigación, de la docencia, y de la misma reputación de las facultades dependerá de su historia y del manejo de las tensiones que estos programas deberán afrontar para su consolidación.

\section{EL ORIGEN}

La creación de los once programas, entre 2004 y $2015^{9}$, hizo parte de un proceso de expansión de los doctorados impulsado por la política pública al nivel nacional, que en estos once años se tradujo en la creación de 4,3 veces más doctorados que los creados en toda la historia del país - pasando de 77 doctorados en 2003 a 329 en 2015 en diferentes

8 Sobre esta cuestión volveremos más adelante.

9 En administración se crearon once en estos años -en Eafit, Universidades de los Andes, Valle, Rosario, EAN (con dos), del Norte, Medellín, Externado, Simón Bolívar y Jorge Tadeo Lozano (en gestión pública)-, a los cuales se deben adicionar dos programas de la Universidad Nacional: uno en ingeniería y otro en economía que cuentan con líneas específicas para administración. Cabe advertir que a comienzos de 2016 se crearon dos nuevos programas: en la Universidad Javeriana de Cali y en el ICESI. De suerte que el país cuenta con 15 programas asociados directamente con la administración. 
campos del saber (SNIES, 2016)-. Por eso, los once apenas representaron el 3,7\% del total creado en el país en esos años, pero constituían "una oleada" en un campo del saber que vio aparecer el primero en 2004 en EAFIT.

Para 2009 esta dinámica empezó a generar preocupación ante los proyectos de creación de doctorados en FCA con poca tradición investigativa y pocos profesores con título de doctor mientras, al mismo tiempo, "se percibía un ambiente permisivo por parte de las autoridades nacionales para la aceptación de programas doctorales de todo tipo y para el ofrecimiento de programas en convenio de toda naturaleza y nivel" (Muñoz, 2016). Con tal intranquilidad como motivador principal, los directores de los programas doctorales que para entonces contaban con registro SNIES -EAFIT, Universidad de los ANDES, Universidad del Rosario, Universidad del Valle y la Universidad Nacional- convinieron conformar la Red de Doctorados en Administración, Dirección y Gestión de Colombia, REDAC, para fortalecer la formación doctoral.

Esta preocupación encuentra respaldo en los estudios de comienzos y fines de los 90 (Rodríguez et al., 1990, 1992; Malaver et al., 2000), que mostraban que la investigación estaba centrada en cuatro Facultades, y que muy pocos grupos y líneas de investigación contaban con trayectorias -tiempo, proyectos y publicaciones- (Calderón y Amaya, 2005) de cuyo desarrollo se desprendiera naturalmente la creación de programas de doctorados. Además, las evidencias sobre la internacionalización de las publicaciones indican que este es un proceso apenas en ciernes y más aún en el caso de los journals Q1 y Q2 (Malaver, 2016). Esto significa que los aprendizajes sobre la divulgación internacional de los conocimientos generados, si bien parecen acelerados, en algunos de estos doctorados parecen corresponder a un learning by doing, que todavía está en curso. Por ello, el desafío para la formación de sus doctorantes parece grande.

\section{DOCTORADOS, INVESTIGACIÓN Y PUBLICACIÓN EN LAS FCA}

Los doctorados potenciarán las capacidades para publicar en las FCA. A la apropiación del estado del arte que supone la publicación internacional, los doctorados adicionan la necesidad de pensar la teoría, de reflexionar sobre los procesos de construcción de la teoría, es decir, la investigación básica y sobre las prácticas teóricas propias, como prerrequisito para garantizar solvencia académica en la oferta de formación de capacidades para investigar al nivel doctoral. Esto es lo que, finalmente, elevará la capacidad de generar conocimiento original y facilitará la elaboración de artículos con mayor nivel de publicabilidad internacional. 
En este sentido, la historia muestra desafíos enormes para esos programas. Sus ofertas formativas se cimientan en los aprendizajes derivados de su experiencia investigativa y de publicación, y los pocos indicios existentes señalan que las publicaciones de los investigadores colombianos - por los años de creación de los programas- estaban basadas en estudios empíricos (Martínez y Páez, 2016). De ahí se colige, aunque solo al nivel de conjetura, que a semejanza de lo ocurrido en Argentina y España, la investigación se ha centrado en la importación y aplicación de la teoría creada en los países desarrollados (Fernández y Gantman, 2011), antes que en el desarrollo -adaptación o creación- del conocimiento.

De cara a la construcción de conocimiento original esa situación demanda cambios profundos en la aproximación a la teoría. Implicaría, por ejemplo, interrogarla desde la realidad con el espíritu crítico que reclama Popper cuando afirma: “trabajamos con teorías, es decir, con sistemas deductivos, y ello por dos razones. En primer lugar una teoría o un sistema deductivo es un intento de explicación, y por consiguiente un intento de solución de un problema. En segundo lugar, una teoría, es decir, un sistema deductivo puede criticarse racionalmente por sus consecuencias. Es así una solución provisional, sujeta a la crítica racional" (Popper, 1994, p. 104); o cuando indica que "si usted está interesado en el problema que yo trato de resolver mediante mi afirmación tentativa, puede ayudarme criticándola lo más severamente que pueda; y si logra idear alguna prueba experimental de la que usted piense que puede refutar mi afirmación, lo ayudaré gustosamente, en lo que de mí dependa, a refutarla" (Popper, 1983, p. 51). Desde la perspectiva de Kuhn, implicaría detectar y confirmar desajustes entre teoría y realidad, y convertirlos ¿por qué no? en fuente de generación de nuevos conceptos o teorías (Kuhn, 1962); pero esto implicaría -en esta perspectiva-, descubrir anomalías en el paradigma y asumirlo. En caso contrario, es hacerle el juego a una trampa estéril, pues "el descubrimiento comienza con la percepción de la anomalía, o sea, con el reconocimiento de que en cierto modo se han violado las expectativas inducidas por el paradigma que rige la época normal" (Kuhn, 1962, p. 93). En síntesis, esos cambios suponen reducir dependencia paradigmática en favor de un mayor diálogo académico, soportado en la generación de conocimiento.

\section{LOS DOCTORADOS EN ADMINISTRACIÓN Y EL MERCADO PARA SUS EGRESADOS}

La inserción laboral de los egresados constituye una fuente -en estado latente- de incertidumbre y tensiones sobre el futuro de los doctorados. Hasta ahora, la expansión de la oferta educativa de administración ha estado ligada al crecimiento del país, que se ha traducido en la ocupación creciente de administradores profesionales que -como se 
señaló antes- ha soportado la proliferación de programas de administración de pregrado y posgrado (1512 en 2016 según el SNIES). Pese a su corta trayectoria, ese vínculo no es claro en el caso de los programas de doctorado. Tensión que Orozco (2016) pone sobre el tapete. Pero, ahondar en esa problemática, de la cual hay solo síntomas, desborda los alcances de este artículo.

Más pertinente es la tensión relacionada con la absorción de los egresados de estos programas al interior de las propias FCA, que constituyen su mercado natural, al menos inicialmente. De acuerdo con Orozco (2016, p. 134) “es necesario investigar si la formación doctoral en Colombia, cuenta con un mercado atractivo para insertar investigadores nacionales o si se valora más la incorporación de doctores de países del Norte que pueden, en principio, incrementar la producción internacional", y sugiere que, en parte, por la presión institucional -las acreditadoras- hay preferencia por los investigadores formados en los países anglosajones (Orozco, 2015), pertenecientes al international club.

Aunque tensionante, esa discusión parece insoslayable. Esta remite a los factores de diferenciación, esto es, al posicionamiento buscado para los egresados de los doctorados de las FCA, en otras palabras ¿qué aspectos específicos de su formación compensaría las desventajas frente a los egresados de programas extranjeros? Superar el déficit de partida supone un desafío enorme para que puedan posicionarse en un mercado que, a poco andar, ya empieza a mostrarse restringido. Para ello, las estrategias pueden variar. Lo invariante debería ser la búsqueda de apuestas efectivas para ofrecer una formación que permita a los egresados generar y divulgar conocimiento con un nivel de calidad (internacional) tal, que les brinde un reconocimiento y una legitimidad académica y social, expresada en los empleos alcanzados.

Esto conduce a discusiones presentes desde mucho antes de la creación de los primeros programas. En la discusión sobre la necesidad de crear programas doctorales propios se planteaba como conveniente evitar que la totalidad de los profesores acometieran los esfuerzos de sus tesis doctorales sobre temas de pertinencia en los países de acogida. Así se evitaría no solo el hecho de que todos los docentes formados como doctores regresaran al país alejados del contexto (Zapata, 1989). Hoy se plantea que los egresados de las universidades internacionales del top tier del management, tienen acceso a redes de conocimiento internacionales y a un mayor conocimiento de las lógicas y dinámicas de la publicación internacional (Orozco, 2016). 
Por eso, es pertinente el escrutinio investigativo sobre las intenciones de las FCA para el ofrecimiento de sus programas de doctorado. Una somera mirada a las declaraciones sobre los propósitos declarados -en sus páginas web-, muestra que apuntan a satisfacer dos demandas, a saber: formar investigadores con capacidad de aportar soluciones prácticas a las dificultades del tejido organizacional del país, y con capacidad para publicar en las más importantes revistas del área sus aportes al conocimiento. Pero atender ambos frentes no es sencillo, de acuerdo con lo discutido en las secciones anteriores sobre las dificultades para la publicación internacional; y los argumentos en torno a que se perdiera la oportunidad de construir redes internacionales relevantes durante su formación doctoral. También se encuentra el argumento opuesto, sobre la descontextualización de los doctorados cursados en el extranjero. Existe, por supuesto, la tercera vía, que es la búsqueda institucional del acceso a redes internacionales de colaboración científica, que parece ser una tendencia en América Latina (Orozco y Villaveces, 2015).

En cualquier caso, si en los artículos que componen este número especial existiera algún nivel de acierto, un desafío que en Colombia se debería asumir es la medición del impacto de los programas de doctorado de las FCA, no solo al nivel de publicaciones y citaciones, sino en la sociedad a la cual se deben las FCA y en el tejido organizacional al que están llamadas a atender para justificar su existencia.

\section{ACADÉMICOS Y PRACTITIONERS EN LAS FCA: UNA CONVERSACIÓN NECESARIA}

La inserción de los doctorados en las FCA es quizá el mayor vector de la "disciplinarización" del conocimiento generado y difundido, pero es fuente de diferencias y tensiones a su interior. El aumento del profesorado con doctorado que reclaman estos programas junto con las demandas de las acreditadoras en tal dirección se traducen en cambios significativos en la composición de la planta docente de las facultades. Por su formación y visión de la academia estos impregnan de teoría y de los resultados de sus investigaciones a los programas de los otros niveles de la oferta educativa de las FCA y esto, poca duda cabe, elevará la calidad de la docencia, especialmente en la formación de posgrados, que requiere de un conocimiento profundo del contexto de aplicación de los conocimientos impartidos. Ese proceso podría dar lugar, incluso, a la configuración de marcos analíticos que faciliten articular los diseños curriculares de los distintos programas ofrecidos.

Pero esto ni es simple ni es fácil de alcanzar. La profundización requerida para la publicación y la teorización puede conducir a una hiper-especialización -monodisciplinar- útil a los intereses individuales de los investigadores, pero que puede dificultar la articulación de 
saberes -como señalaba Pfeffer (1993)-, que es consustancial al carácter interdisciplinar de la administración y a una oferta educativa de calidad y, al contrario, puede dar lugar a una fragmentación de los contenidos que es a todas luces perjudicial. Excepto para el investigador que haya convertido el medio -la publicación- en fin.

Otra tensión, la existente entre los profesores con perfiles más académicos y más de practitioners, que ya era reconocida por los pioneros de los estudios sobre la investigación en las FCA, persistirá pues es consustancial al management. El reto es entonces cómo lograr una coexistencia más productiva. En principio, quienes propugnan por tender puentes entre los dos, como Bartunek y Rynes (2014), aceptarían que desde la perspectiva de los académicos, con la teoría se podría "lleva más rigor a la práctica" (Simon, 1967) y, como plantea Mintzberg (2005), se podrían ampliar los marcos de referencia de los directivos empresariales, y desde allí “cuestionar sus paradigmas y prácticas gerenciales", para transformarlas; mientras que con el concurso de los practitioners, se podrían "llevar los problemas de la realidad al aula". Pero dado que son como "agua y aceite", sin una adecuada gestión de dicha tensión en las FCA, ambos tienden a repelerse (Simon, 1967).

Por lo visto, la gestión de esas tensiones incidirá en la dinámica y consolidación de los doctorados y, por esta vía, en el ritmo y dirección de la disciplinarización y de la generación de conocimiento en las FCA. A su vez, esto dependerá de la gestión al interior de las facultades del diálogo -interdisciplinar- entre sus académicos, y entre estos y los practitioners. Ahora bien, los pocos indicios existentes en el país sobre los procesos de creación y desarrollo de los doctorados en el management sugieren que los desafíos para manejar estas tensiones también son enormes.

\section{Balances y reflexiones}

El rápido recorrido efectuado aquí por más de cinco décadas en la evolución de la investigación sobre la administración en Colombia, hecho a partir de las evidencias y análisis de los estudios sobre esa investigación, aporta elementos -algunas de cuyas implicaciones aquí se discuten- que contribuyen al logro de una visión amplia y comprensiva del presente y de las alternativas para su consolidación futura.

Una mirada de conjunto a la trayectoria de la investigación en las FCA muestra, por una parte, que su tardío inicio y su lento avance, que se prolongó hasta mediados de los años 90 y que fue caracterizado como un persistente estado incipiente, se debió básicamente a obstáculos en las FCA y, por otra, que el doble quiebre -histórico- en la trayectoria que 
provocaron, primero, la institucionalización y despegue de la investigación y, luego, la internacionalización -en ciernes- de sus publicaciones fueron inducidos por la política de ciencia, tecnología e innovación y por la presión de fuerzas institucionales externas a las FCA. Hacia el futuro, esa mirada permite prever una consolidación de la investigación -con la internacionalización de la publicación y el desarrollo de los programas de doctorado como sus puntas de lanza-, cuyo ritmo, dirección y alcances dependerá del manejo que den las FCA a las tensiones y desafíos consustanciales a los procesos en curso.

El entorno ha tenido un papel importante y positivo en el avance de la administración en el país. Los procesos de industrialización y la competencia desatada por la apertura económica y la globalización parecen haber generado la demanda que soportó el surgimiento de las FCA y la proliferación de programas de formación en administración en el país al nivel de pregrado y posgrado. Y ahora, las políticas y fuerzas institucionales han incidido significativamente en la institucionalización y la previsible consolidación de la investigación. Pero a futuro podrían restringir esa dinámica. Por ello, parece necesario para las FCA responder a la presión institucional y, a la vez, generar dinámicas internas para trascenderlas.

Así lo indica una comparación de las dinámicas y orientaciones que han guiado la investigación en el ámbito internacional y nacional. Entre los años 60 y 90 la investigación internacional en el management avanzaba -a grandes pasos- guiada por el modelo de las ciencias naturales y como vía escogida para alcanzar la excelencia académica y elevar su estatus en el mundo académico, mientras en Colombia, por el contrario, se luchaba por su existencia y se abogaba por desarrollar conocimiento que ayudara a dar respuesta a los problemas nacionales. Pero ahora, mientras en los países desarrollados se cuestionan los sesgos y efectos negativos del modelo asumido desde los años 60 , y se pide un mayor equilibrio entre rigor y relevancia, las vías deductivas e inductivas, los métodos cuantitativos y cualitativos, la valoración de la contribución a la teoría y la utilidad social de la misma, y tender puentes entre académicos y practitioners (Corley y Gioia, 2011; Bartunek y Rynes, 2014), aquí, al contrario, bajo el influjo de una internacionalización inducida por la presión institucional, la evaluación se centran en el impacto medido por citaciones, que privilegia la contribución académica y desincentiva la investigación motivada por su utilidad social. Aplicarlos, sin más, significaría un retroceso de décadas frente a los avances en la discusión internacional.

De ese modo, el acelerado proceso de internacionalización de los parámetros para evaluar el conocimiento generado -y publicado-, inducidos por Colciencias, acreditadoras 
y rankings, al contrastarse con el estado de la discusión internacional muestra que, para el caso de la administración, tales parámetros -basados en la citación-son insuficientes para dar cuenta de lo que, por su naturaleza, hoy exige este campo del saber al nuevo conocimiento generado: originalidad y utilidad práctica (Corley y Gioia, 2011), es decir, influyente para los académicos y útil al nivel empresarial y social. En consecuencia, para captar el impacto y superar los sesgos de la citación, que hoy se intentan superar en el ámbito de la administración, se deben generar nuevas métricas e indicadores sobre el uso social de las publicaciones (Malaver, 2016). Así, una dosis de autonomía sería pertinente y saludable.

La mirada a las tendencias internacionales también muestra la vigencia del propósito de convertir la realidad propia en fuente de conocimiento relevante, original y en potencial fuente de diálogo académico entre los países desarrollados y emergentes. Se reconoce que las diferencias en los niveles de desarrollo, culturas, valores, visiones e instituciones, originan prácticas y problemas administrativos cuya explicación puede requerir de nuevos conceptos o teorías (Barkema et al., 2015). Frente a los avances que están sucediendo en Europa y Asia (Augier y Sullivan, 2007; Sanabria et al., 2014; Barkema et al., 2015), se considera "disfuncional" el pretendido universalismo científico, que es más bien un vestigio del parroquialismo de la comunidad académica norteamericana (Augier, March y Sullivan, 2005).

En esta dirección, avanzar implica superar la mera replicación del conocimiento importado que parece existir en Latinoamérica (Fernández y Gantman, 2011; Ibarra, 2006); reconocer el valor central del contexto -donde se centran 0campo, Gentilin y Gonzáles (2016)-, recuperar y retroalimentar, en síntesis, la fuerza con que se planteaba en décadas anteriores la necesidad de generar conocimiento más original para resolver los problemas del país (Dávila, 1988; Echeverry et al., 1990). Necesidad expuesta, por fortuna, en algunos estudios sobre la dinámica actual de la investigación (Sanabria et al., 2014; Calderón et al., 2010) y los artículos publicados en este Especial plantean tal inquietud. Queda por indagar si esa preocupación es transversal a los artículos que hoy se originan en las FCA y se publican internacionalmente.

La "oleada" de creación de doctorados en las FCA, registrada en la última década, refuerza los procesos de institucionalización de la investigación y de internacionalización de las publicaciones y, al mismo tiempo, agencia el tránsito de la profesionalización a la disciplinarización en la producción y difusión del conocimiento al interior de las FCA. 
Pero el cumplimiento de su papel enfrenta grandes desafíos en ambos frentes. En el primer caso, el pensar la teoría y su construcción, para formar con solvencia a los doctorantes, debería ser fuente de generación de conocimiento original publicable en revistas de alto impacto; pero esto plantea acelerados aprendizajes para la mayoría de estos doctorados, dadas las cortas trayectorias investigativas y experiencia en la publicación internacional de alta calidad existente en sus inicios; tarea esencial para lograr el posicionamiento de los programas y forjar capacidades en sus egresados que faciliten su adecuada inserción laboral. En el segundo caso, cabe esperar que los doctorados a través de sus docentes irradien el resto de programas de pregrado y posgrado, llevando más rigor a la práctica, y ayudando a configurar marcos analíticos que faciliten la articulación curricular, pero pueden conducir a una hiper-especialización, que dificulte el diálogo académico y genere fragmentación de los contenidos ofrecidos. El diálogo entre académicos y entre estos y los practitioners que, es indispensable para consolidar la disciplinarización y ofertas educativas de calidad, solo sería posible con una adecuada gestión de dichas tensiones.

\section{Limitaciones e investigaciones necesarias}

La intención que anima este texto es aportar insumos para una discusión académica sobre las perspectivas de avance de la investigación en las FCA. Por ello, sólo busca mostrar los rasgos más generales de la trayectoria de la investigación y de los factores que han definido su ritmo y dirección, de manera que ayude a entender el presente y los desafíos y alternativas de futuro. Por ello, no aborda los temas con la profundidad y detalle requerido para concluir. El objetivo del artículo consiste en abrir espacios para el diálogo e invitar al debate académico. Y de allí derivan sus mayores limitaciones, aquí reconocidas y asumidas.

Por ello, algunos temas centrales y asociados con la problemática abordada, y que ameritan ser estudiados a profundidad, no fueron discutidos o se mencionaron tangencialmente. Uno es el tema de las conversaciones. Si el avance del conocimiento puede entenderse como el resultado de una conversación, y si como nos recuerdan 0campo et al. (2016, p. 15 "toda conversación está condicionada por el contexto de los interlocutores al momento de construir el discurso", es de alto interés establecer si entre los autores vinculados a las FCA hay algún nivel de conversación en algún tema y si éste tiene como referente su propio contexto y si, en consecuencia, este diálogo ha construido algún entendimiento sobre las organizaciones y prácticas administrativas colombianas; si hay creación y acumulación de conocimiento colectivo sobre el país, en otros términos, si hay algún nivel de construcción de comunidad académica. Es útil discutir, incluso, si hay necesidad de ella o si en virtud de la internacionalización es una alternativa anacrónica. 
En el contexto de la discusión sobre la investigación, otro tema vital es establecer el papel de las revistas académicas editadas en las FCA. Las cuales, por lo visto en Malaver (2016), han jugado un papel central en el proceso de internacionalización en ciernes, especialmente las admitidas en la WoS y Scopus, es decir, que siguen las lógicas de los journals top. Allí cabría preguntarse, por ejemplo, si su papel es solo servir de trampolín para la internacionalización; o si desde otra perspectiva, igual que las otras revistas indexadas en sistemas latinoamericanos, -como Latindex, Scielo, RedAlyc- estarían llamadas a servir de vehículo para articular comunidades académicas responsables de la construcción de conocimiento que revele las particularidades de nuestra realidad, como paso necesario para ampliar el conocimiento internacional existente. En cualquiera de los dos casos, en la dinámica imperante, las revistas cuya existencia y esfuerzos están fuera de WoS y Scopus son y serán invisibilizadas, al igual que el conocimiento que divulgan. Y esto representa un descomunal desperdicio de recursos, esfuerzos y conocimiento social.

\section{Referencias}

AACSB International. (2012). Impact of research. A guide for Business Schools. The Association to Advance Collegiate, Schools of Business. Tampa, Florida.

Alvesson, M., and J. Sandberg. (2013). Has management studies lost its way? Ideas for more imaginative and innovative research. Journal of Management Studies, 50 (1), 128-152.

Augier, M. and J. March. (2007). The pursuit of relevance in a management education. California Management Review, 49 (3), 129-143.

Augier, M.; J. March, and B. Sullivan. (2005). Notes on the evolution of a research community: organization studies in Anglophone North America, 1945-2000. Organization Science, $16(1), 85-95$.

Barkema, H.; X-P. Chen, G. George, Y. Luo, and A. Tsui. (2015). West meets East: New concepts and theories. Academy of Management Journal, 58 (2), 460-479.

Bartunek, J.M., and S.L. Rynes. (2014). Academic and practitioners are alike and unlike: Paradoxes of academic-practitioner relationships. Journal of Management, 40 (5), 1181-1201.

Bennis, W.G., and J. 0'Toole. (2005). How business schools lost their way. Harvard Business Review, 83 (5), 96-104.

Calderón, G. y M. Amaya. (2005). La investigación en administración en Colombia: una descripción a partir de los grupos reconocidos por Colciencias. En Calderón y Castaño (Comps.). Investigación en Administración en América Latina: evolución y resultados (pp. 48-53). Manizales: Universidad Nacional de Colombia.

Calderón, G.; A. Castaño, L. García y E. Castro. (2014). Generación de conocimiento en los grupos élite de investigación en Colombia. Manizales: Universidad Nacional de Colombia. 
Calderón, G.; J. Arrubla, L. Gutiérrez, G. Castaño, R. Posada, A. Ruiz, H. Serna y J. Vivares. (2010). La investigación en administración en Colombia. Condiciones para la generación de conocimiento, investigadores, institucionalización, y producción científica. Bogotá: Ascolfa.

Chanlat, A.; R. Echeverry y C. Dávila. (1990). Hacia una administración propia para América Latina? En R. Echeverry, C. Davila y A. Chanlat (eds.) En búsqueda de una administración para América Latina: experiencias y desafios (pp. 13-32). Cali: Universidad del Valle.

Colquit, J.A., and C.P. Zapata. (2007). Trends in the theory testing: A five decade study of the "Academy of Management Journal". Academy of Management Journal, 50 (6), 1281-1303.

Corley, K.G., and D.A. Gioia. (2011). Building theory about theory building: What constitutes a theoretical contribution? Academy Management Review, 36 (1), 12-32.

Dávila, C. (1980). La crisis de la educación en Administración en Colombia. Revista Eafit Temas Administrativos, 39, 20-35.

Dávila, C. (1982). Lineamientos y experiencias sobre una política de investigación en las escuelas de administración de América Latina. Memorias II del Encuentro de Investigadores en Administración. ICFES, Universidad del Valle, 3 al 6 de marzo, Cali.

Dávila, C. (1988). La investigación en administración: barreras y perspectivas. Academia Revista Latinoamericana de Administración, 1, 1-19.

Dávila, C. (1990). A propósito del informe Porter Mckibbin: anotaciones comparativas sobre los estudios de administración en Estados Unidos y América Latina. En R.D. Echeverry, C. Dávila y A. Chanlat (eds.) En búsqueda de una administración para América Latina: Experiencias y desafios (pp. 155-164). Cali: Universidad del Valle.

Dávila, C. (1991). Ensayos sobre la educación en administración en Colombia. Monografías. Uniandes, Serie Educación y Administración, $N^{\circ} 24$.

Dávila, C. (2005). Generación de conocimiento administrativo en América Latina: ¿realidad, necesidad o utopía? En Calderón y Castaño (comps.) Investigación en Administración en América Latina: evolución y resultados (pp. 39-43). Manizales: Universidad Nacional de Colombia. Dinero (2000). Las mejores Escuelas de negocios. Revista Dinero, 101, 24-44.

Echeverry, R.; A. Chanlat y C. Dávila. (1990). (comps.) En búsqueda de una administración para América Latina: experiencias y desafíos. Cali: Universidad del Valle.

Fernández, C.J.; and E. Gantman. (2011). Spain and Argentina as importers of management knowledge (1955-2008): A comparative analysis. Canadian Journal of Administrative Science, 28, 160-173.

George, G. (2014). Rethinking management scholarship. Academy of Management Journal, 57 (1), 1-6.

Gordon, R.A.; and J.E. Howell. (1959). Higher Education for Business. New York, Columbia University Press. 
Hambrick, D.C. (2007). The field of management's devotion to theory: Too much of a good thing? Academy of Management Journal, 50, 1346-1352.

Ibarra, E. (2006). Organization studies and epistemic coloniality in Latin America: Thinking otherness from the margins. Organization, 13 (4), 463-488.

Kuhn, T. (1962). The structure of scientific revolutions. Chicago: Universidad de Chicago.

López, F. (1998). Educación en administración y modas administrativas en Colombia. Revista Universidad EAFIT, 109, 59-88.

López, F. (2001). El surgimiento de EAFIT en el contexto del desarrollo de la educación en administración y la práctica administrativa en Colombia. Revista Universidad EAFIT, 121, 9-18.

Malaver, F. (1998). La formación del recurso humano y la competitividad empresarial en Colombia. Revista EAN, 33-34, 5-17.

Malaver, F. (1999). La investigación en gestión empresarial. Academia - Revista Latinoamericana de Administración, 23, 67-81.

Malaver, F. (2006). El despegue de la investigación colombiana en Administración: análisis de sus avances en el periodo 2000-2006. Cuadernos de Administración, 18 (32), 71-109.

Malaver, F. (2016). La internacionalización de la publicación colombiana en el campo de la administración: avances y desafíos. Cuadernos de Administración, 29 (52), 53-81.

Malaver, F.; L. Romero, M. Cortes, J. Ruíz, J. Perdomo y G. Peralta. (2000). Investigación en gestión empresarial: ¿proceso naciente? Colombia, 1965-1998. Bogotá: Corporación Calidad y Colciencias.

Martínez, M. y I. Páez. (2016). Orientaciones estratégicas de los investigadores en el campo de la administración: un estudio comparativo entre regiones y disciplinas. Cuadernos de Administración, 29 (52), 83-114.

Mayor, A. (1990). La profesionalización de la administración de empresas en Colombia. En búsqueda de una administración para América Latina: Experiencias y desafios. (pp. 99-109). Cali: Universidad del Valle.

Mintzberg, H. (2005). Directivos, no MBAs. Una visión crítica de la dirección de empresas y la formación empresarial. España: Universidad de Deusto.

Muñoz, R. (2016). Presentación ante pares académicos para acreditación alta calidad EAFIT. Informe interno.

Ocampo, C. A.; M. Gentilin y D. R. Gonzáles. (2016). Conversaciones sobre administración y organizaciones en Latinoamérica. Un énfasis en el estado actual de la investigación y la educación. Cuadernos de Administración, 29 (52), 13-51.

Ogliastri, E. (1982). Investigaciones en Administración. Memorias, II Encuentro de investigadores en Administración. Cali: ICFES, Universidad del Valle. 
Orozco, L.A. (2015). Diversidad y heterogeneidad en redes de colaboración científica. Un estudio de las escuelas de administración de América Latina. Bogotá: Universidad Externado de Colombia.

Orozco, L.A. (2016). La formación doctoral en administración en Colombia: reflexión fenomenológica de un investigador. Cuadernos de Administración, 29 (52), 115-139.

Orozco, L.A., and J.L. Villaveces. (2015). Heterogeneous research networks in Latin American Schools of business management. Academia, Revista Latinoamericana de Administración, 28 (1), 115-134.

Pfeffer, J. (1993). Barriers to the advance of organizational science: Paradigm development is a dependent variable. Academy Management Review, 18 (4), 599-620.

Pfeffer, J., and C.T. Fong. (2001). The end of business schools? Less success than meets the eye. Academy of Management Learning and Education, 1 (1), 78-95.

Pierson, F.C. (1959). The Education of American Businessmen: A Study of University-College Programs of Business Administration. New York: McGraw Hill.

Popper, K. (1983). Conjeturas y refutaciones. Barcelona: Paidos.

Popper, K. (1994). Tolerancia y responsabilidad intelectual. En búsqueda de un mundo mejor. Barcelona: Paidos.

Rafols, I.; L. Leydesdorff, A. O'Hare, P. Nightingale, and A. Stirling. (2012). Howjournal rankings can suppress interdisciplinary research: A comparison between innovation studies and business \& management. Research Policy, 41, 1262-1282.

Rodríguez, M.; C. Dávila y L. Romero. (1990). Estado actual y perspectivas de la educación y la investigación en el área de Administración. En Misión de Ciencia y Tecnología. En búsqueda de una administración para América Latina: Experiencias y desafios (pp. 155-164). Cali: Universidad del Valle.

Rodríguez, M.; C. Dávila y L. Romero. (1992). Gerencia privada, gerencia pública, educación en crisis. Bogotá: Universidad de los Andes.

Sanabria, M.; J. Saavedra y A. Smida. (2014). La construcción de sentido de los investigadores en gestión en Colombia acerca de su campo de conocimiento. Innovar, 24 (54), 7-24.

Simon, H.A. (1965). Administrative behavior. A study of decision making in administrative organization. New York: Free Press.

Simon, H.A. (1967). The business Schools. A problem in organizational design. Journal of Management Studies, 4 (1), 1-16

Sistema Nacional de Información de la Educación Superior - SNIES, (2016). http://snies.mineducacion.gov.co/consultasnies/programa, Ministerio de Educación Nacional, Consultado en junio de 2016.

Zapata, A. (1989). Memorias primer encuentro de doctorados en América Latina. Cali: Universidad del Valle. 